\title{
BMJ Open Understanding and responding to the cost and health impact of short-term health staffing in remote and rural Aboriginal and Torres Strait Islander community-controlled health services: a mixed methods study protocol
}

\author{
Michelle S Fitts (D) , ${ }^{1}$ John Humphreys, ${ }^{2}$ Terry Dunbar, ${ }^{3}$ Lisa Bourke, ${ }^{4}$ \\ Edward Mulholland, ${ }^{5}$ Steven Guthridge, ${ }^{6}$ Yuejen Zhao (i) , ${ }^{7}$ Michael P Jones, ${ }^{8}$ \\ John Boffa, ${ }^{9}$ Mark Ramjan, ${ }^{10}$ Lorna Murakami-Gold, ${ }^{11}$ Annie Tangey, ${ }^{12}$ \\ Clarissa Comerford, ${ }^{12}$ Rosalie Schultz, ${ }^{12}$ Narelle Campbell, ${ }^{13}$ Supriya Mathew, ${ }^{1}$ \\ Zania Liddle, ${ }^{1}$ Deborah Russell, ${ }^{1}$ John Wakerman ${ }^{1}$
}

To cite: Fitts MS, Humphreys J, Dunbar T, et al. Understanding and responding to the cost and health impact of short-term health staffing in remote and rural Aboriginal and Torres Strait Islander community-controlled health services: a mixed methods study protocol. BMJ Open 2021;11:e043902. doi:10.1136/ bmjopen-2020-043902

- Prepublication history for this paper is available online. To view these files, please visit the journal online (http://dx.doi org/10.1136/bmjopen-2020043902).

Received 19 August 2020 Accepted 05 May 2021

A) Check for updates

(c) Author(s) (or their employer(s)) 2021. Re-use permitted under CC BY-NC. No commercial re-use. See rights and permissions. Published by BMJ.

For numbered affiliations see end of article.

\section{Correspondence to}

Professor John Wakerman; john.wakerman@menzies. edu.au

\section{ABSTRACT}

Introduction Access to high-quality primary healthcare is limited for remote residents in Australia. Increasingly, remote health services are reliant on short-term or 'fly-in, fly-out/ drive-in, drive-out' health workforce to deliver primary healthcare. A key strategy to achieving health service access equity, particularly evident in remote Australia, has been the development of Aboriginal Community Controlled Health Services (ACCHSs). This study aims to generate new knowledge about (1) the impact of short-term staffing in remote and rural ACCHSs on Aboriginal and Torres Strait Islander communities; (2) the potential mitigating effect of community control; and (3) effective, context-specific evidence-based retention strategies.

Methods and analysis This paper describes a 3-year, mixed methods study involving 12 ACCHSs across three states. The methods are situated within an evidencebased programme logic framework for rural and remote primary healthcare services. Quantitative data will be used to describe staffing stability and turnover, with multiple regression analyses to determine associations between independent variables (population size, geographical remoteness, resident staff turnover and socioeconomic status) and dependent variables related to patient care, service cost, quality and effectiveness. Qualitative assessment will include interviews and focus groups with clinical staff, clinic users, regionally-based retrieval staff and representatives of jurisdictional peak bodies for the ACCHS sector, to understand the impact of short-term staff on quality and continuity of patient care, as well as satisfaction and acceptability of services.

Ethics and dissemination The study has ethics approval from the Human Research Ethics Committee of the Northern Territory Department of Health and Menzies School of Health Research (project number DR03171), Central Australian Human Research Ethics Committee (CA-19-3493), Western Australian Aboriginal Health Ethics Committee (WAAHEC-938) and Far North Queensland Human Research

\section{Strengths and limitations of this study}

- This will be the first empirical examination of the use and impact of short-term staffing in rural and remote Aboriginal Community Controlled Health Services (ACCHSs).

- This research project is designed as a partnership and has been shaped and approved by partnering ACCHSs, with implementation strongly guided by the steering committee and the Aboriginal and Torres Strait Islander research team members.

- Recognising and respecting the importance of local skills and knowledge, trained interpreter/translators and community-based researchers will be employed to enable participants to speak in their language of preference and provide guidance on cultural protocols.

- Rigorous evidence generated from the study can inform policy and more effective practice that will help to stabilise the remote health workforce, save money and ultimately, contribute to 'closing the gap' in health outcomes.

- Although the findings may be relevant to similar remote and regional ACCHSs, the findings may not be generalisable to all remote and regional ACCHSs.

Ethics Committee (HREC/2019/QCH/56393). Results will be disseminated through peer-reviewed journals, the project steering committee and community/stakeholder engagement activities to be determined by each ACCHS.

\section{BACKGROUND}

Mortality rates and burden of disease rise with increasing distance from major cities across Australia. ${ }^{1}$ Access to health service 
declines with increasing remoteness, and consequently rates of preventable admissions to hospital increase markedly with remoteness. ${ }^{2}$ While conceptualisation of access differs between theoretical frameworks, many of them do agree that access relates to characteristics of health service providers and the characteristics and expectations of the patient, the dynamic interaction between supply (location, availability or cost of services) and demand (the burden of disease and knowledge, attitudes and skills and self-care practices of the population). ${ }^{3-5}$

The need for an accessible and effective primary healthcare (PHC) system is widely recognised as key to improving health outcomes for Aboriginal and Torres Strait Islander communities and closing the gap in health outcomes. ${ }^{6}$ Aboriginal and Torres Strait Islander Australians living in remote areas experience unacceptably high rates of injury, chronic disease and poverty. ${ }^{7}$ Despite these health needs, the availability of a stable resident health workforce which can deliver effective PHC services is frequently lacking in rural and remote communities. ${ }^{8-10}$

At the same time, a key strategy to achieving equity of access to appropriate and effective health services in Australia has been the development of over 140 Aboriginal Community Controlled Health Services (ACCHSs) over the past 50 years. ACCHSs range in size, infrastructure and workforce. ACCHSs, like other Aboriginal organisations, have a governance model where the service is operated by, and accountable to, the local Aboriginal community. ACCHSs contribute to improving the health and well-being of Aboriginal and Torres Strait Islander peoples through several pathways, including communitycontrolled governance, providing employment and training, strengthening the broader health system and providing accessible, comprehensive PHC. ${ }^{11}$

A growing body of evidence indicates that remote and rural population access to healthcare is not simply about geographical distance and availability of health professionals. Appropriate models of care meeting local population needs are equally important. ${ }^{9}$ Serving one-third to one-half of the Aboriginal and Torres Strait Islander population, ${ }^{12}$ many ACCHSs demonstrate an exemplary model of comprehensive PHC specifically shaped for local Aboriginal and Torres Strait Islander communities. A small but significant body of research from Australia and Canada demonstrates an association between community control, and improved access and health outcomes. ${ }^{113-16}$

PHC workforce models in remote Australia differ from those in urban and rural settings. ${ }^{17}$ Remote clinics usually consist of small teams comprising resident remote area nurses (RANs) and Aboriginal and Torres Strait Islander Health Practitioners (ATSIHPs) supported by visiting general practitioners, specialist doctors and allied health professionals. ATSIHPs operate as independent practitioners, working alongside and collaboratively with other clinicians including doctors, nurses, midwives, allied health and oral health practitioners in a range of settings. ${ }^{18}$ In their role, they may have a broad individual clinical scope of practice or work in a more specialised role, depending on their qualification, clinical setting, level of supervision and community need.

In Australia, the transition of government-run PHC services to community control is supported by both national ${ }^{19}$ and state governments. ${ }^{20}$ Within ACCHSs, two in three $(65 \%)$ positions are held by Aboriginal or Torres Strait Islander peoples ${ }^{21}$ and as many as $93 \%$ of patient consults involve ATSIHPs. ${ }^{22}$ In communities with ACCHSs, family connections between resident health staff and their communities contribute to increased participation in health activities ${ }^{23}$ and community members having a sense of ownership of the service ${ }^{24}$; The presence of people from the local community, employment of Aboriginal and Torres Strait Islander people in the service are key contributors to improved accessibility. ${ }^{25}$

For over a decade, there has been an increasing reliance on a short-term or 'fly-in, fly-out'/'drive-in, drive-out' (FIFO/DIDO) health workforce to fill vacant positions and overcome the health workforce distribution problems in remote Australia. ${ }^{26}$ The substantial increase in a FIFO/DIDO health workforce has been supported by government in the form of funding for the Remote Area Health Corps, which provides short-term employment in the Northern Territory (NT) averaging from 3 to 4 weeks per placement. ${ }^{27}$ There has been a concomitant proliferation of private staffing agencies contributing to this workforce trend. ${ }^{28} \mathrm{~A}$ recent cross-sectional analysis in the NT found that $42 \%$ of remote nurses employed by NT Department of Health were employed on a casual or agency con tract. $^{29}$

The FIFO/DIDO model potentially provides benefits, such as providing coverage while permanent staff take annual and other types of leave and participate in professional development offsite. Short-term staff may provide 'fresh eyes' and have different perspectives to offer in remote clinics. ${ }^{30}$ Furthermore, those short-term staff who have familiarity with specific communities from previous work in those communities, may require little orientation and may bring with them a broad range of knowledge and depth of experience that may otherwise not be available in the community. The team recently completed a project with similar aims and methods with remote NT Government clinics, ${ }^{31}$ finding tensions between the benefits and disadvantages of using FIFO/DIDO staff. ${ }^{30}$ For example, there was a clear hierarchy between agency and permanent RANs, where permanent staff reproduced specific team cultures and ways of practising.

The heavy reliance on short-term staff, who move from community to community or work in communities for short periods and then leave, has also raised significant concerns about the impacts on patient care, on resident health service staff and on health system costs. ${ }^{32}$ Health workforce stability may be less of a problem in ACCHSs because of employment of local community members and Aboriginal and Torres Strait Islander peoples, stronger community links and Aboriginal and Torres Strait Islander governance. Better availability and continuity of care with ATSIHPs in ACCHSs, for example, may help compensate 
for lack of continuity of nurses and other primary care providers. ${ }^{33}$ However, there is a dearth of empirical data relating specifically to the impact of community control on workforce retention.

The costs of maintaining agency and short-term staffing are high, with the costs proportional to staff turnover and remoteness. In the previous project with NT Government clinics, the team quantified high usage of agency nurses, declining usage of ATSIHPs and an alarmingly high rate of staff turnover in remote NT Government-run clinics. ${ }^{8}$ Remote nurse annual turnover rates, for example, average $148 \%$ per annum. Turnover rates of ATSIHPs are almost half this rate $(79 \%)$ and may be even lower in remote ACCHSs. ${ }^{8}$ We have also quantified the considerable costs of high staff turnover in government-run clinics. Reducing remote resident staff turnover to $40 \%$ per annum (which is still very high by international standards) would result in savings of \$A21 million per annum for primary care in NT Government remote clinics. ${ }^{34}$ The analyses also demonstrated the significant cost-ineffectiveness of high turnover and agency staff usage, with potential savings of \$A32 million per annum to the NT health system if staff turnover were halved. ${ }^{35}$ Sustainable employment of local community members is key to improved access to culturally safe care in government-run clinics. ${ }^{30}$

\section{Aims}

Given the importance of providing accessible, sustainable and appropriate PHC in remote and rural Aboriginal and Torres Strait Islander communities, the aims of this study are to generate new knowledge about:1) the impact of short-term staffing in remote and rural ACCHS's on Aboriginal communities ;2) the potential mitigating effect of community control; and, 3) effective, contextspecific evidence-based retention strategies.

The study will compare the effects of two distinct PHC governance models and thereby contribute to the development of evidence-based workforce strategies to stabilise the remote and rural health workforce and improve access to essential PHC services for Aboriginal and Torres Strait Islander peoples in rural and remote areas. The hypotheses to be tested are that:

1. In ACCHSs, high utilisation of short-term health staff has a negative impact on resident health teams in remote and rural Aboriginal and Torres Strait Islander communities in terms of increased workload and stress, decreased professional satisfaction and decreased length of stay;

2. In ACCHSs, high turnover and high utilisation of short-term health staff in remote and rural Aboriginal and Torres Strait Islander communities have a negative impact on the quality, acceptability, safety and continuity of patient care;

3. High turnover and high utilisation of short-term health staff increases service costs and decreases effectiveness of ACCHSs in remote and rural Aboriginal and Torres Strait Islander communities;
4. Community control has a mitigating effect on utilisation of short-term staff and on staff turnover. These in turn have an impact on service effectiveness, cost and consumer and staff satisfaction in remote Aboriginal and Torres Strait Islander communities. Hence, we predict a lesser impact of short-term staff utilisation on these parameters in ACCHSs when compared with NT Government-run PHC services.

\section{METHODS/DESIGN}

This mixed methods study will benefit from our experience working with government-run PHC services. ${ }^{31}$ Using similar, refined methods will enable important comparisons to be made between NT Government-led remote health services and ACCHSs regarding the use and impact of short-term staff. Using a mixed methods approach will help ensure that data are rich and interpreted appropriately. A systematic review will also form part of this programme of work. ${ }^{36}$ Building on earlier systematic reviews, ${ }^{3738}$ this review will investigate the associations between interventions to retain health workers working in rural and remote areas of high-income countries and retention (Hypothesis 4).

\section{Setting and location}

The study will be implemented in August 2019 and finish in August 2022. The project will be conducted in partnership with 12 remote and rural ACCHSs located in NT, Western Australia (WA) and Queensland. Participating services represent a range of jurisdictions and service sizes. Rural locations are within remoteness area (RA) categories RA3 and RA4; remote locations are RA5 as defined by the Australian Statistical Geography Standard-Remoteness Area taxonomy. ${ }^{39}$ The remote Aboriginal and Torres Strait Islander communities and townships that these ACCHSs service range in size from small localities of around 100 people to large remote communities of approximately 3000 people. ${ }^{40}$

\section{Conceptual framework}

The study uses a results-based logic model, adapted for the Australian context, which links health service resource inputs, outputs and outcomes with policies, programmes or initiatives (see figure 1). ${ }^{41} 42$ PHC inputs (eg, funding arrangements, infrastructure linkages and workforce), and PHC delivery through three categories of work processes (governance and public participation, healthcare leadership and management and clinical activities and decisions) are intended to produce specific outputs (eg, products and services) and are the primary link in the chain through which outcomes are achieved. ${ }^{42}$ There are three types of direct PHC effectiveness outcomes: (1) immediate (direct), (2) intermediate (indirect) and (3) final. Immediate outcomes are those for which the PHC workforce, including policymakers and practitioners, can reasonably assume control, responsibility and accountability. Intermediate outcomes include areas in which PHC stakeholders have a lesser degree of control, but for 


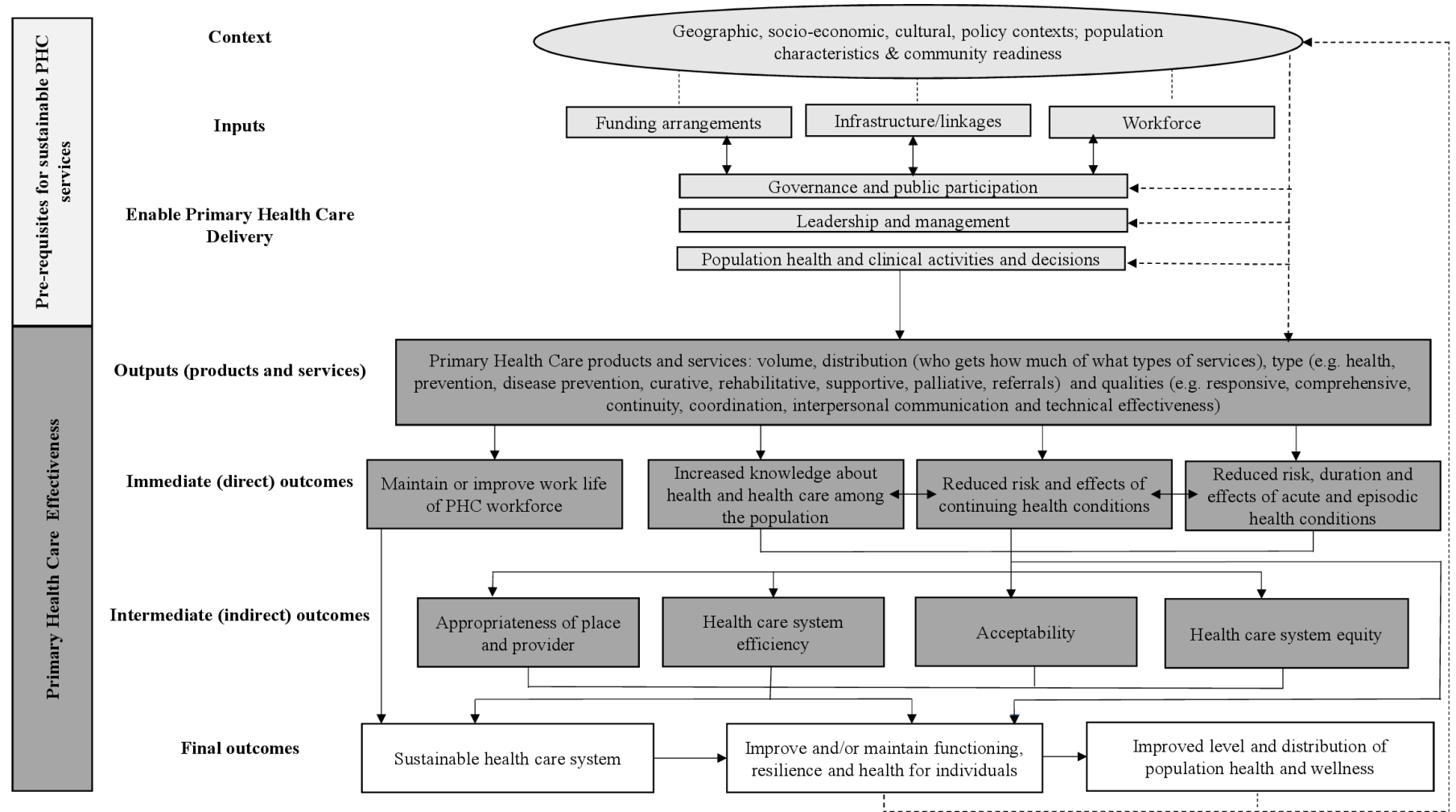

Figure 1 An evidence-based logic model for primary healthcare for small rural and remote communities. PHC, primary healthcare.

which PHC services are still expected to have an impact. Final outcomes are long-term PHC objectives for individuals, the population and the healthcare system.

Conceptually, this study will address how a specific context characterised by supportive policy (with respect to community control) and change from government control to structural community control ("community readiness' $)^{43}$ influences the inputs and enablers (ACCHSs' governance, leadership, workforce). These inputs will be linked to immediate (direct), intermediate (indirect) and to final outcomes. Using this logic model ensures that appropriate data are collected to inform all components of the research, and that the contribution of context, inputs and enablers are monitored against relevant outcome measures. The research will contribute to the health workforce literature through generating new knowledge about how context, inputs, enablers and outcomes are linked.

\section{Quantitative methods and analysis \\ Data}

The study will use retrospective quantitative data that pertain to both individuals and clinics for the period from 2010 to the latest data available in 2020. Specifically, the study will use routinely collected administrative data sets including hospitalisations, death registries, primary care utilisation (at participating ACCHSs and government-run remote clinics), National Aboriginal Health Key Performance Indicators (nKPIs), financial data and payroll/employment data. In order to monitor workforce issues and trends, the research will also include documentation collected by ACCHSs such as annual staff job satisfaction surveys, exit interviews and organisational charts. To accurately attribute hospitalisations to ACCHS clients, data linkage methods will be used to link ACCHSs' client records with hospital separation minimum data sets, death registry data and primary care utilisation at government-run clinics and aggregated for each study-month.

Data and key information from ACCHSs will also be collected systematically to develop profiles for each participating service. This data collection tool will be developed in conjunction with the ACCHSs and piloted with several ACCHSs prior to being administered. Data variables will include changes over time in number of staff positions by profession and site, timing and nature of retention interventions, service population, geographical footprint, number and names of clinics, data management processes, format in which data can be provided, capability of providing different data items, uniformity across clinics within each ACCHS (eg, in management systems) and measures of geographical remoteness including Accessibility Remoteness Index of Australia Plus and distances from nearest hospital and nearest city. ${ }^{44}$ These data will be collected from a range of sources including publicly available information on the internet (eg, Australian Bureau of Statistics data), privately provided data (eg, Accessibility Remoteness Index of Australia Plus) and through email and telephone calls between researchers and the key contact person at each ACCHS. 
Data linkage is planned for linking individual-level primary care data from ACCHSs (where available) to hospitalisation data. Third party data linkage organisations in each jurisdiction will provide the data linkage service (eg, SA-NT Datalink for ACCHSs whose patients are admitted to NT hospitals; WA Data Linkage Branch for the ACCHSs whose patients are admitted to WA hospitals; Research Linkage Group at Queensland Health for the ACCHSs whose patients are admitted to Queensland hospitals).

\section{Statistical analysis}

Personnel and finance data provided by ACCHSs will be used to determine staff exits from clinics and calculate staff turnover rates, stability rates, use of casual, agency and locum staff and staffing full-time equivalents (FTEs) during each clinic-month. These will be the major measures of inputs as per the logic model in figure 1 .

Correlations between use of casual/agency/locum staff and staff turnover/stability measures will provide an initial indication of impact of short-term health staff on resident health teams (Hypothesis 1). Statistical associations between casual/agency/locum staff and permanent staff FTEs will be further investigated using univariate $\chi^{2}$ analysis with total and linear trend decomposition of $\chi^{2}$ values for the linear correlation coefficient. ${ }^{45}$ Multivariate analysis will use both dynamic and autoregressive Markov switching models for the total FTEs as the dependent variable with turnover, stability and casual/agency/locum staff proportion as non-switching covariates.

Correlations between measures of staff turnover/ stability (key independent variables) with measures of quality of care such as nKPIs and potentially preventable hospital admissions (dependent variables) will provide a further indication of impact of short-term health staffing on quality of care (Hypothesis 2). Associations will be additionally tested using general linear models (linearisation method) to estimate within-clinic correlation and adjust SEs accordingly. The nKPIs will be expressed as sequential change from the previous period (month, quarter or half year) to express change in indicator status. Direction and strength of associations will be indicated by $\beta$ coefficients.

Two cost-effectiveness analyses will compare the costeffectiveness of clinics with higher staff turnover or higher use of casual/agency/locum staff with clinics with lower turnover or lower use of casual/agency/locum staff, respectively (Hypothesis 3). Incremental costeffectiveness ratios will be calculated with key primary care effectiveness proxies being hospitalisation rates and years of life lost (YLL) rates, respectively. Total hospitalisation and YLL rates have been used as acceptable proxy measures for the effectiveness of PHC in the costeffectiveness literature.

Costs per person-month will be calculated using ACCHSs' expenditure and hospitalisation data. Jurisdictional average costs per hospitalisation will be sought and used as benchmark prices for hospitalisations. A threshold of \$A120000 (2020 value, adjusted for inflation in other years) will be used as the benchmark price for a YLL. Cost-effectiveness planes and cost-effectiveness acceptability curves will be examined to investigate uncertainty of incremental cost-effectiveness ratios and acceptability of savings per hospitalisation and per YLL.

Measures of staff turnover/stability, clinical utilisation, service effectiveness, quality and cost will be compared between ACCHSs and government-run clinics using data collected from a previous project ${ }^{31}$ using general linear models with SEs adjusted for repeated measures (Hypothesis 4). We will also track changes in staffing patterns, clinical utilisation, quality of care and costs in clinics which have transitioned from government-run to community control within the period under consideration.

In all analyses, statistical inference will employ the nonparametric bootstrap method to deal with violations of the assumption of normality (calculation of $\mathrm{p}$ values). All analyses will adjust for potential confounders such as community population size, clinical size and geographical remoteness.

\section{Qualitative methods \\ Participants}

Purposeful and convenience qualitative recruitment methods will be used to recruit participants to the study. Interviews and focus groups will be conducted with the following four groups of participants:1) staff of ACCHSs, 2)clinic users, 3)regional centre-based specialists and retrieval staff and, 4)jurisdictional peak bodies for the ACCHS sector. Large and small clinics (determined by staff FTE) in rural and remote communities will be selected for in-depth analysis. The fieldwork is proposed to be completed between February 2020 and December 2021. Table 1 describes the eligibility criteria, sample size, data collection method, consent and proposed topics to be explored across all participant groups.

\section{Recruitment}

Staff of ACCHSs

Like in the previous project with NT Government clinics, ${ }^{3046}$ interviews will be held in locations that are convenient and appropriate to each participant. Creating safe and comfortable space can aid the promotion of privacy and need for confidentiality for clinical staff and researchers during the interviews. At the clinics, the interviews will be carried out in a quiet room away from the clinical areas and removed from disturbance, so participants are able to talk freely about their perceptions and experiences.

\section{Clinic users}

Local Aboriginal community-based research assistants (CBRAs) will be employed in each community to assist with promotion of the project within the community, participant recruitment as well as interview and focus group facilitation. Translators or CBRAs will assist with translation for those participants for whom English is not 


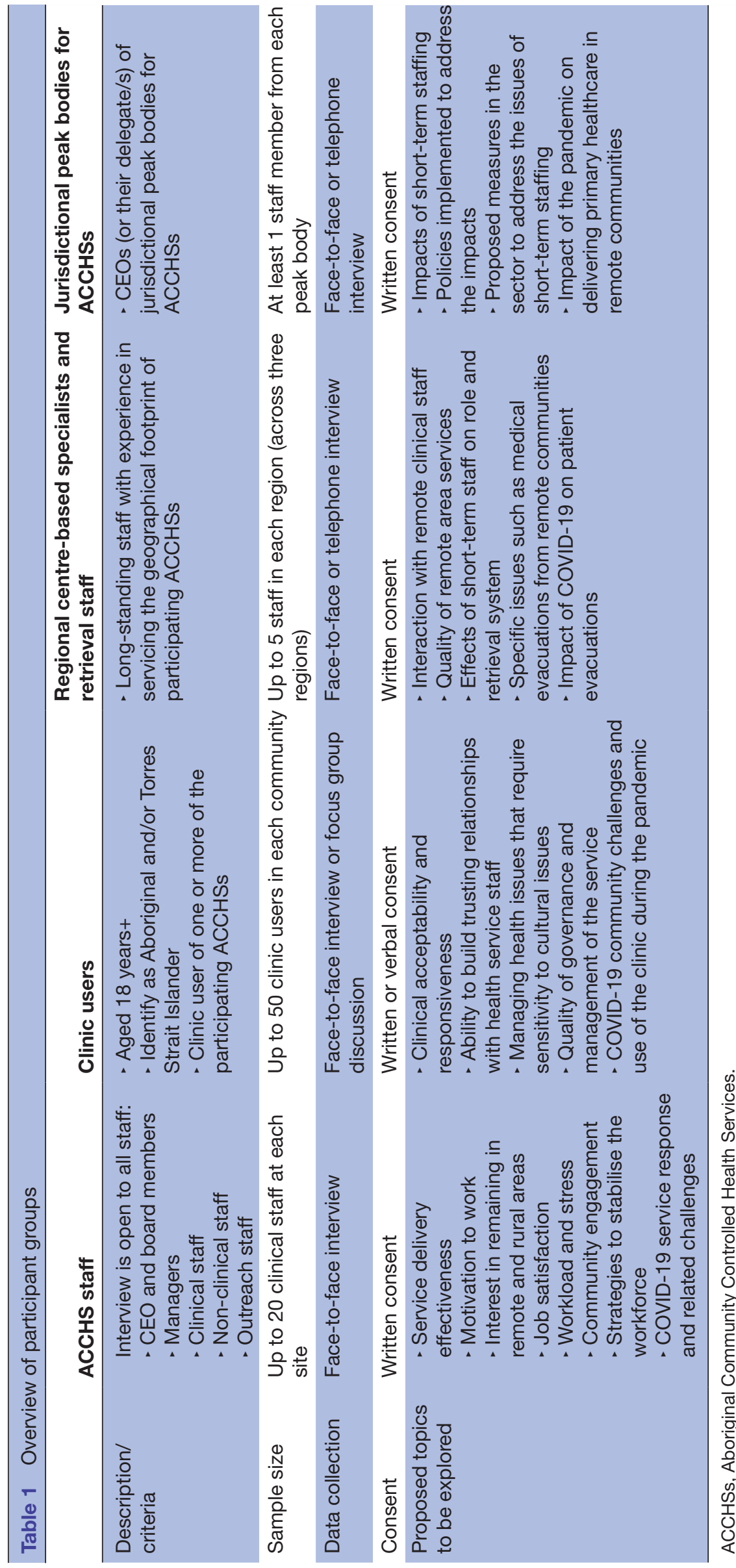


their first language. Individualised training will be delivered to CBRAs to ensure they have a full understanding of the project and ethical processes to recruit participants as well as increase their qualitative skills to undertake focus group sessions with Aboriginal and Torres Strait Islander community members. Where possible one male and one female CBRA will be employed to work as part of the research team in each community. Each CBRA will receive a certificate acknowledging the training provided and demonstrated skills practised during the research project which may increase future research opportunities.

To ensure a variety of clinic users are recruited, the research team will implement diverse data collection activities including: 1. meeting with groups run by existing services; 2. other events such as paid stalls at local shopping centres or festivals; 3. a stall at participating clinics; and 4. going door-to-door with the CBRAs. The research team will work closely with community leaders, clinics and councils to recruit clinic users. Specifically, the research team will seek direction regarding promotion and recruitment methods prior to each visit. Promotion and recruitment of clinic users may differ between communities .

\section{Regional centre-based specialists and retrieval staff}

Regional centre-based specialist and retrieval staff will be recruited to participate in an interview. Participants in the regional areas will be recruited through the local hospital or ACCHS networks. Regional centre-based specialists and retrieval staff will be approached through usual health service communication channels for their interest in being interviewed.

\section{Jurisdictional peak bodies for the ACCHS sector}

CEOs (or their delegate/s) of jurisdictional peak bodies for ACCHSs in NT, WA and Queensland will be recruited through usual organisational communication channels.

Participants from all groups will be provided with an information sheet prior to providing informed consent and being interviewed.

\section{Sample size}

\section{Staff of ACCHSs}

Participation in an interview will be open to all staff including clinical, non-clinical and outreach staff. All short-term staff (eg, casuals, agency staff and locum tenens staff) present at the time of fieldwork will also be invited to be interviewed. There is no minimum amount of time staff have to have been employed by the service to participate in the study. Recruitment will continue at each site until thematic saturation is reached. In clinics with small numbers of staff, all consenting staff will be recruited. Due to resourcing and access challenges, staff who are no longer employed at partnering ACCHSs will not be approached to participate in the study.

\section{Clinic users}

To ensure a variety of perspectives are collected, in each town or remote community, up to 50 clinic users will be invited to participate in the study or until thematic saturation is reached. To reach saturation on a certain topic, a minimum of four focus groups is required. ${ }^{47}$ Data saturation will be checked after each meeting. We aim for at least five participants per focus group. If there are fewer than five participants, the dynamics and the interaction of the group will be limited. If there are more than 10 participants, it is likely there will not be enough time to let every participant express their view. It can be difficult to determine how many people will be available and willing to participate in the study. When conducting the focus groups, age, gender and family group will be accounted for to ensure a safe space for open discussion. Clinic users will have the opportunity to 'tell their story' around the personal, family and community impacts of short-term staffing and high turnover of staff.

\section{COVID-19 pandemic}

There will be inherent limitations in relation to the data we can collect as well as new challenges facing services to maintain their workforce during the fieldwork. ${ }^{48}$ Given the uncertainties associated with the COVID-19 pandemic, it is essential that the research team remains responsive and flexible, provides meaningful information to participating ACCHSs and is able to adapt recruitment processes. To achieve this, changes have been made to the data collection and recruitment processes. Additional questions have been added to the interview protocols for all participant groups. For example, ACCHSs staff will also be asked about the impact of the pandemic on recruiting and retaining staff, workload challenges, availability of resources (eg, personal protective equipment), changes in community use of the clinic and responses by the clinic and health service to respond to these challenges (eg, telemedicine). Clinic users will also be asked about the impact of COVID-19 on their day-to-day life (eg, delays/forgoing cultural practices; food availability), use of clinical services in the pandemic environment, what they know about COVID-19 and what resources they have received.

Additionally, research team members have implemented guidelines for conducting remote community fieldwork which align and comply with organisational and government guidelines and recommendations. To minimise potential risks of spreading COVID-19, events that bring together large groups of people (eg, barbeques) will be precluded. To ensure the research team models safe practices (eg, social distancing), the team will hold small focus groups (eg, up to five participants). Where possible, these focus group discussions will be held outdoors. For focus group discussions held with existing groups run by non-government agencies and service providers, the research team will comply with any additional guidelines. At events where the team is promoting and recruiting clinic users in public spaces, COVID-19 information will be displayed along with hand hygiene products. The project's COVID-19 guidelines will be regularly reviewed and 
updated accordingly to organisational and government guideline changes.

\section{Data analysis}

The interviews and focus groups will be audiotaped with consent, and transcribed verbatim. If recording is not consented, handwritten notes will be taken during the non-audio recorded interview. Information about participants' identity will be removed. Interview transcripts and written notes will be managed using NVivo V.12 (QSR International), a qualitative software package designed to assist analysing large amounts of text-based data.

Inductive thematic analysis as described by Braun and Clarke $^{49}$ will be conducted to capture the stories of how the FIFO/DIDO workforce has shaped health and healthcare in the community. This approach involves coding statements based on their key concepts, combining these coded concepts into themes and refining the identified themes. Common themes will be identified among clinical staff, rural centre-based specialists and retrieval staff, staff of jurisdictional peak bodies for ACCHS separately from clinical users of the ACCHS clinics. The coding will be reviewed by the research team and investigators before the data are thematically analysed. Aboriginal and Torres Strait Islander investigators and research team staff will be involved in all aspects of the qualitative data coding and analysis to ensure that a cultural lens is applied in the coding and analysis of data. ${ }^{50}$ We will also compare the qualitative findings from ACCHSs with the qualitative findings from the remote NT Government-run clinics from a previous project. ${ }^{31}$

\section{Patient and public involvement}

Patients and/or members of the public were not involved in the development of the research questions and design of the study. The results of the study will be disseminated to study participants through activities and pathways to be determined by each participating ACCHS (as described in knowledge translation section below).

\section{Ethics and dissemination \\ Ethical considerations}

The study has ethics approval from the Human Research Ethics Committee of the Northern Territory Department of Health and Menzies School of Health Research (project number DR03171), Central Australian Human Research Ethics Committee (CA-19-3493), Western Australian Aboriginal Health Ethics Committee (WAAHEC-938) and Far North Queensland Human Research Ethics Committee (HREC/2019/QCH/56393).

Trained interpreters and CBRAs will be employed to assist the research team with explaining the project and participant information sheet in the local language and translating during interviews and focus group discussions. All participants will provide written consent and will be free to withdraw at any time. No identifying information will be kept with the data, which will be analysed and stored on a secure network drive at the research institution, protected by password and used only for the purposes of this study. Only the researchers involved in this study will have access to the quantitative and qualitative data. No information about the project will be published in any form that will allow any individual to be recognised, with non-identifiable results presented in reports and publications.

\section{Project governance}

This study is designed as a partnership and has been shaped and approved by the participating ACCHSs and Aboriginal and Torres Strait Islander leaders. The project will establish a steering committee consisting of CEOs (or their delegates) of the ACCHSs participating in the study and the Aboriginal Medical Services Alliance NT to guide the research team to effectively achieve the project objectives (data provision, analysis, reporting, dissemination and turning the results into policy and practice). Specifically, the steering committee will provide guidance on, contribute to and formally approve key decisions relating to research design, data collection (including both field work to collect primary data and collection of secondary administrative data), data analysis and interpretation. Members of the committee will assist with facilitating requests for and approvals needed to access service data locally and with the relevant state/territory authorities. Results will be communicated to the steering committee, ensuring that Aboriginal and Torres Strait Islander leaders will contribute to formulating recommendations in relation to policy and practice and play a key role in communicating and disseminating results within their own communities and the broader Australian community. The committee will also have an important role in determining relevant retention strategies to be implemented to improve workforce retention in ACCHSs. The group will meet regularly, with individual contact between the research team and group members arranged as needed. The research team also consists of Aboriginal and Torres Strait Islander researchers who will have full participation in the implementation, analysis and documentation of the study.

\section{Knowledge translation}

Research translation is an integrated feature of the project. With extensive experience in research translation, ${ }^{52}$ the research team will implement a multifaceted translation strategy. It will be informed by both the quantitative component of the study along with the qualitative interviews to determine current and potential strategies to stabilise the health workforce. Researchers will work with all partnering ACCHSs and the steering committee to jointly interpret the project findings and ensure that they are disseminated in meaningful and sensitive ways to service staff and community members.

The research team will deliver presentations to national conferences and to smaller forums; meet with senior policymakers in Aboriginal and Torres Strait Islander Health at Federal and State/Territory levels; and author 
peer-reviewed publications. This translation strategy has been highly successful in the recent NT Government study conducted by the research team, ${ }^{31}$ which has included invitations to rural and executive level meetings to present study results and to propose solutions, including improved training pathways, more flexible workforce conditions, retention incentives and improved targeting of suitable remote health staff. Findings regarding costs, quality, service effectiveness and staff motivation will be presented to the steering committee. Recommendations will also be made about ongoing workforce retention monitoring systems that are relevant to context. This will occur through annual face-to-face meetings, teleconference meetings and through regularly emailed one-page updates.

Each ACCHS will be provided with summary and comparative data for like services (while maintaining confidentiality for each participating ACCHS). Information about publications will also be provided to ACCHSs as a plain language summary with infographics to support their internal research translation activities. The CEOs and steering committee members will ensure that the outcomes of the project will be disseminated to the participants. The processes by which this occurs will be at the discretion of the ACCHSs' CEOs and boards.

\section{DISCUSSION}

This project aims to generate rigorous new knowledge by analysing data collected from ACCHSs, their staff and by the participating ACCHSs. The evidence generated related to costs, quality, effectiveness and the impact of community control will help partnering services to understand more about effective strategies to maintain a strong rural and remote health workforce. The findings may also have applicability to PHC models in nations with similarly colonised Indigenous populations, including in Aotearoa/New Zealand, Canada and the United States of America. ${ }^{53-55}$

Underpinned by a results-based logic model, adapted for the Australian context, ${ }^{41}{ }^{42}$ this project will enable a better understanding of the complex interplay between health service delivery models in remote Aboriginal and Torres Strait Islander communities, PHC workforce patterns and healthcare costs and health outcomes. In areas where there is high unmet need, additional PHC resources may result in enhanced diagnosis of conditions and complications, and potentially an increase in health system contact including hospitalisation. There may be a non-linear relationship between PHC utilisation and hospitalisations ${ }^{56}$ but there is considerable international ${ }^{57}$ and local ${ }^{58}$ evidence that optimising PHC access results in decreased hospitalisations, improved health outcomes and, importantly, overall decreased costs. For example, Zhao et $a l^{59}$ conservatively estimated savings to the NT Government of \$A125 million annually with improved access to PHC in remote NT communities.
Given previously demonstrated high rates of remote staff turnover in the government sector, there are likely substantial potential savings also to be made in the non-government sector. ${ }^{34}$ The research team will work with members of the steering committee and key policymakers, to use information about costs, quality, service effectiveness and motivation to develop a set of strategies that have the potential to address workforce turnover and maldistribution. The research findings will contribute towards understanding how services can improve access and enhance service effectiveness of PHC services in remote and rural Australia.

The knowledge generated from this research is essential to closing the gap in health outcomes for Aboriginal and Torres Strait Islander Australians. ${ }^{6}$ The reasons Aboriginal and Torres Strait Islander Australians living in remote and rural areas have poor health outcomes compared with non-Indigenous Australians are complex. ${ }^{17}$ Colonisation and ongoing discrimination, racism, intergenerational trauma and, for some, disconnection to culture and country have contributed to a high burden of disease. ${ }^{59-61}$ These inequalities such as racism can have significant impacts on access to healthcare ${ }^{61}$ Furthermore, limited healthcare access and utilisation contributes to persistent health inequality and poor health outcomes. ${ }^{5962}$ For example, there is an association between PHC utilisation and potentially preventable hospital admissions. ${ }^{59}$ Synthesis of existing international evidence indicates that ACCHSs can increase access to healthcare for Aboriginal and Torres Strait Islander peoples, ${ }^{63}$ though the extent to which this is the case is not well understood; nor is there strong evidence about the impact of health workforce turnover, retention and supply issues on access to health services provided by ACCHSs. The new knowledge generated by this study will continue to fill this evidence gap.

Notwithstanding its many strengths, this study is not without potential limitations. Key among these are issues with quality and completeness of data that can be expected as high staff turnover and short retention may impact on quality of clinical data entry and data management in participating ACCHSs. Health service administrative systems may also have changed during the study period, rendering data for earlier parts of the study inaccessible. Our study also uses proxies for quality of primary care, including a range of clinical process and outcome measures routinely collected and reported as nKPIs. There are recognised limitations with some indicators, including defining Indigenous regular clients by the number of visits in the past 2 years rather than by their usual address in the health service catchment, recency of introduction of some indicators for reporting and time trend anomalies for example, related to changes in cervical screening methods. ${ }^{64}$ 
Author affiliations

${ }^{1}$ Menzies School of Health Research, Charles Darwin University, Alice Springs, Northern Territory, Australia

${ }^{2}$ School of Rural Health, Monash University, Strathdale, Victoria, Australia ${ }^{3}$ Indigenous Social and Wellbeing Centre, School of Population Health Research, Australian National University, Canberra, Australian Capital Territory, Australia ${ }^{4}$ Department of Rural Health, The University of Melbourne, Shepparton, Victoria, Australia

${ }^{5}$ Miwatj Health Aboriginal Corporation, Nhulunbuy, Northern Territory, Australia ${ }^{6}$ Centre for Child Development and Education, Menzies School of Health Research, Casuarina, Northern Territory, Australia

${ }^{7}$ Northern Territory Department of Health, Darwin, Northern Territory, Australia

${ }^{8}$ Psychology Department, Macquarie University, North Ryde, New South Wales, Australia

${ }^{9}$ Central Australian Aboriginal Congress, Alice Springs, Northern Territory, Australia

${ }^{10}$ Top End Health Service, Northern Territory Government, Casuarina, Northern

Territory, Australia

${ }^{11}$ Poche SA + NT, Flinders University, Alice Springs, Northern Territory, Australia

${ }^{12}$ Ngaanyatjarra Health Service, Alice Springs, Northern Territory, Australia

${ }^{13}$ Flinders Northern Territory, College of Medicine and Public Health, Flinders University, Darwin, Northern Territory, Australia

Acknowledgements We acknowledge the time and contribution of the study participants and the staff from participating Aboriginal Community Controlled Health Services.

Contributors MSF drafted and revised the manuscript. JW conceived the study, contributed to design and reviewed the manuscript. JH contributed to the conceptualisation and design of the study and assisted with drafting the manuscript. LB and TD contributed to the design of the study, particularly the qualitative component, and provided comments on the manuscript. MPJ, SG, YZ and $\mathrm{DR}$ contributed to the design of the study, particularly the quantitative component, and provided comments on the manuscript. NC, JB, EM, MR, AT, CC, RS and LM-G contributed to the design of the study. DR, SM and ZL contributed to the drafting of the manuscript. All authors read and approved the final manuscript.

Funding The project is supported under the Australian Research Council's Project Discovery funding scheme (project number DP190100328) and by the Medical Research Future Fund through the NHMRC and Central Australian Academic Health Science Network (N/A). The information and opinions contained in it do not necessarily reflect the views or policy of the Commonwealth of Australia (or the Department of Health).

Competing interests None declared.

Patient and public involvement Patients and/or the public were not involved in the design, or conduct, or reporting, or dissemination plans of this research.

Patient consent for publication Not required.

Provenance and peer review Not commissioned; externally peer reviewed.

Open access This is an open access article distributed in accordance with the Creative Commons Attribution Non Commercial (CC BY-NC 4.0) license, which permits others to distribute, remix, adapt, build upon this work non-commercially, and license their derivative works on different terms, provided the original work is properly cited, appropriate credit is given, any changes made indicated, and the use is non-commercial. See: http://creativecommons.org/licenses/by-nc/4.0/.

\section{ORCID iDs}

Michelle S Fitts http://orcid.org/0000-0003-2839-5430

Yuejen Zhao http://orcid.org/0000-0002-5775-4503

\section{REFERENCES}

1 Australian Institute of Health and Welfare. Australia's health 2018. Australia's health series no. 16. AUS 221. Canberra, ACT: AlHW, 2018.

2 Council of Australian Governments Reform Council. Healthcare 2011-12: comparing performance across Australia. Canberra: COAG Reform Council, 2013.

3 Penchansky R, Thomas JW. The concept of access: definition and relationship to consumer satisfaction. Med Care 1981;19:127-40.

4 Levesque J-F, Harris MF, Russell G. Patient-Centred access to health care: conceptualising access at the interface of health systems and populations. Int J Equity Health 2013;12:18.
5 Gulliford M, Figueroa-Munoz J, Morgan M, et al. What does 'access to health care' mean? J Health Serv Res Policy 2002;7:186-8.

6 Holland C. Close the gap 2018 - a ten-year review: the closing the gap strategy and recommendations for reset. Canberra, ACT: The Close the Gap Campaign Steering Committee, 2018.

7 Vos T, Barker B, Begg S, et al. Burden of disease and injury in Aboriginal and Torres Strait Islander peoples: the Indigenous health gap. Int J Epidemiol 2009;38:470-7.

8 Russell DJ, Zhao Y, Guthridge S, et al. Patterns of resident health workforce turnover and retention in remote communities of the Northern Territory of Australia, 2013-2015. Hum Resour Health 2017:15:52-12.

9 Wakerman J, Humphreys JS, Wells R, et al. Features of effective primary health care models in rural and remote Australia: a casestudy analysis. Med J Aust 2009;191:88-91.

10 Wakerman J, Humphreys J, Russell D, et al. Remote health workforce turnover and retention: what are the policy and practice priorities? Hum Resour Health 2019;17:99

11 Campbell MA, Hunt J, Scrimgeour DJ, et al. Contribution of Aboriginal community-controlled health services to improving Aboriginal health: an evidence review. Aust Health Rev 2018;42:218-26.

12 Dwyer JM, Lavoie J, O'Donnell K. Contracting for Indigenous health care: towards mutual accountability: contracting for Indigenous health care. Aust J Public Adm 2011;70:34-46.

13 Bath J, Wakerman J. Impact of community participation in primary health care: what is the evidence? Aust J Prim Health 2015;21:2.

14 Lavoie JG, Forget EL, Prakash T, et al. Have investments in on-reserve health services and initiatives promoting community control improved first nations' health in Manitoba? Soc Sci Med 2010:71:717-24.

15 O'Neil J, Gallagher J, Wylie L, et al. Transforming first Nations' health governance in British Columbia. Int $J$ Health Gov 2016;21:229-44.

16 Panaretto KS, Wenitong M, Button S, et al. Aboriginal community controlled health services: leading the way in primary care. Med $J$ Aust 2014;200:649-52.

17 Wakerman J. Innovative rural and remote primary health care models: what do we know and what are the research priorities? Aust $J$ Rural Health 2009;17:21-6.

18 Queensland Health. Aboriginal and Torres Strait Islander health practitioner: scope of practice guidelines, 2008. Available: https:// www.health.qld.gov.au/_data/assets/pdf_file/0019/731251/ATSIHP_ scope-practice-guideline.pdf

19 Australian Government. National Aboriginal and Torres Strait Islander health plan 2013-2023. Canberra, ACT: Commonwealth of Australia, 2013.

20 Northern Territory Aboriginal Health Forum. Pathways to community control: an agenda to further promote Aboriginal community control in the provision of primary health care services. Darwin, NT: Northern Territory Government, 2008.

21 Baeza JI, Lewis JM. Indigenous health organizations in Australia: connections and capacity. Int J Health Serv 2010;40:719-42.

22 Larkins SL, Geia LK, Panaretto KS. Consultations in general practice and at an Aboriginal community controlled health service: do they differ? Rural Remote Health 2006;6:560.

23 Taylor J, Dollard J, Weetra C, et al. Contemporary management issues for Aboriginal community controlled health services. Aust Health Rev 2001;24:125-32.

24 Freeman T, Baum F, Lawless A, et al. Case study of an Aboriginal community-controlled health service in Australia: universal, RightsBased, publicly funded comprehensive primary health care in action. Health Hum Rights 2016;18:93-108.

25 Gomersall JS, Gibson O, Dwyer J, et al. What Indigenous Australian clients value about primary health care: a systematic review of qualitative evidence. Aust N Z J Public Health 2017;41:417-23.

26 Zhao Y, Russell DJ, Guthridge S, et al. Long-term trends in supply and sustainability of the health workforce in remote Aborigina communities in the Northern Territory of Australia. BMC Health Serv Res 2017:17:836-10.

27 Studdert L. Remote area health Corps: nurses making a contribution to primary health services in the NT. Australas EMerg Nurs $J$ 2010;13:142.

28 Allen \& Clarke. Evaluation of the child health check initiative and the expanding health service delivery initiative: summary report. Canberra, ACT: Department of Health and Ageing, 2011.

29 Northern Territory Department of Health. Remote area nurse safety on-call after hours security. Darwin, NT: Northern Territory Government, 2016

30 Bourke L, Dunbar T, Murakami-Gold L. Discourses within the roles of remote area nurses in Northern Territory (Australia) government- 
run health clinics. Health Soc Care Community 2020. doi:10.1111/ hsc.13195. [Epub ahead of print: 12 Oct 2020].

31 Wakerman J, Humphreys J, Bourke L, et al. Assessing the impact and cost of short-term health workforce in remote Indigenous communities in Australia: a mixed methods study protocol. JMIR Res Protoc 2016;5:e135.

32 House of Representatives Standing Committee on Regional Australia. Cancer of the bush or Salvation for our cities? Fly-in, flyout and drive-in, drive-out workforce practices in regional Australia. Canberra, ACT: Parliament of Australia, 2013.

33 Pulver LJ, Fitzpatrick S, Ritchie J. Filling the gap: an evaluation of a voluntary dental program within an Aboriginal and Torres Strait Islander community controlled primary health service. Aborig IsI Health Work J 2010;34:3-7.

34 Zhao Y, Russell DJ, Guthridge S, et al. Cost impact of high staff turnover on primary care in remote Australia. Aust Health Rev 2019;43:689-95.

35 Zhao Y, Russell DJ, Guthridge S, et al. Costs and effects of higher turnover of nurses and Aboriginal health practitioners and higher use of short-term nurses in remote Australian primary care services: an observational cohort study. BMJ Open 2019;9:e023906.

36 Hines S, Wakerman J, Carey TA. Retention strategies and interventions for health workers in rural and remote areas: a systematic reviewer protocol. JBI Database Syst Rev Implement Rep 2020;18:89-96.

37 Buykx P, Humphreys J, Wakerman J, et al. Systematic review of effective retention incentives for health workers in rural and remote areas: towards evidence-based policy. Aust $J$ Rural Health 2010;18:102-9.

38 Dolea C, Stormont L, Braichet J-M. Evaluated strategies to increase attraction and retention of health workers in remote and rural areas. Bull World Health Organ 2010;88:379-85.

39 Department of Health. Australian statistical geography standard - remoteness area. Canberra: Australian Government, 2020. https://www.health.gov.au/health-workforce/health-workforceclassifications/australian-statistical-geography-standardremoteness-area

40 Australian Bureau of Statistics. Census 2016. Canberra, ACT: ABS, 2016.

41 Watson DE, Broemeling A-M, Wong ST. A results-based logic model for primary healthcare: a conceptual foundation for population-based information systems. Healthc Policy 2009;5 Spec no:33-46.

42 Watson DE, Broemeling AM, Reid R, et al. A Results-Based logic model for primary health care: laying an evidence-based Foundation to guide performance measurement, monitoring and evaluation. Vancouver, BC: Centre for Health Services and Policy Research, 2004.

43 Baum F. The new public health. Melbourne: Oxford Unversity Press, 2016.

44 Australian Bureau of Statistics. The Australian statistical geography standard (ASGS) remoteness structure (ASGC), 2020. Available: http://www.abs.gov.au/websitedbs/D3310114.nsf/home/ remoteness+structure

45 Armitage P, Berry G, Matthews JNS. Statistical methods in medical research. 4th ed. Oxford: Wiley-Blackwell, 2008.
46 Dunbar T, Bourke L, Murakami-Gold L. More than just numbers! perceptions of remote area nurse staffing in Northern Territory government health clinics. Aust $J$ Rural Health 2019;27:245-50.

47 Crabtree BF, Millers WL. Doing qualitative research. 2 nd ed. USA: Sage, 1999.

48 Fitts MS, Russell D, Mathew S, et al. Remote health service vulnerabilities and responses to the COVID-19 pandemic. Aust $J$ Rural Health 2020;28:613-617.

49 Braun V, Clarke V. Using thematic analysis in psychology. Qual Res Psychol 2006;3:77-101.

50 Barnett L, Kendall E. Culturally appropriate methods for enhancing the participation of Aboriginal Australians in health-promoting programs. Health Promot J Austr 2011;22:27-32.

51 Kowal E, Anderson I, Bailie R. Moving beyond good intentions: Indigenous participation in Aboriginal and Torres Strait Islander health research. Aust N Z J Public Health 2005;29:468-70.

52 Buykx P, Humphreys J, Wakerman J, et al. 'Making evidence count': a framework to monitor the impact of health services research. Aust $J$ Rural Health 2012;20:51-8.

53 Ellison-Loschmann L, Pearce N. Improving access to health care among New Zealand's Maori population. Am J Public Health 2006;96:612-7.

54 Lavoie JG, Dwyer J. Implementing Indigenous community control in health care: lessons from Canada. Aust Health Rev 2016;40:453-8.

55 Kunitz SJ. The history and politics of US health care policy for American Indians and Alaskan natives. Am J Public Health 1996;86:1464-73.

56 Griffiths K, Coleman C, Lee V, et al. How colonisation determines social justice and Indigenous health - a review of the literature. $J$ Popul Res 2016;33:9-30.

57 Zhao Y, Wright J, Guthridge S, et al. The relationship between number of primary health care visits and hospitalisations: evidence from linked clinic and hospital data for remote Indigenous Australians. BMC Health Serv Res 2013;13:466.

58 Starfield B, Shi L, Macinko J. Contribution of primary care to health systems and health. Milbank Q 2005;83:457-502.

59 Zhao Y, Thomas SL, Guthridge SL, et al. Better health outcomes at lower costs: the benefits of primary care utilisation for chronic disease management in remote Indigenous communities in Australia's Northern Territory. BMC Health Serv Res 2014;14:463.

60 Gracey M, King M. Indigenous health part 1: determinants and disease patterns. Lancet 2009;374:65-75.

61 Awofeso N. Racism: a major impediment to optimal indigenous health and health care in Australia'. Australian Indigenous HealthBulletin 2011;11:1-15.

62 Carson B, Dunbar T, Chenhall R, et al. Social determinants of Indigenous health. NSW: Allen \& Unwin, 2007.

63 Harfield SG, Davy C, McArthur A, et al. Characteristics of Indigenous primary health care service delivery models: a systematic scoping review. Global Health 2018:14:12-1.

64 Australian Insittuite of Health and Welfare. 2017 national key performance indicators for Aboriginal and Torres Strait Islander primary health care: results from June 2016. National key performance indicators for Aboriginal and Torres Strait Islander primary health care series No. 4. cat. No. IHW 177. Canberra, ACT: Australian Insittuite of Health and Welfare, 2017. 